\title{
MULTISCALE MODELING OF FRC COMPOSITES
}

\author{
Jithender J. Timothy, Yijian Zhan AND Günther Meschke \\ Ruhr University Bochum \\ Bochum, Germany \\ e-mail: timothy.jithenderjaswant@rub.de,yijian.zhan@rub.de \& guenther.meschke@rub.de
}

Key words: micromechanics, frc, composites, element fragmentation, lefm, material design, multiscale

\begin{abstract}
We propose a multiscale model for FRC composites that is a combination of semianalytical and computational sub-models specified at multiple scales. At the scale of the single fiber, a semi-analytical model is developed that characterizes the microslip behavior at the interface between the matrix and the fiber in terms of the overall composite stresses. The influence of fiber bundles on microcrack bridging and arrest is taken into account within the framework of the linear elastic fracture mechanics. Upscaling to the macroscopic level is achieved by using continuum micromechanics. We show that the macroscopic deformation of the FRC composite is governed by a 'TERZHAGI' like effective stress. Selected numerical experiments provide insight into the role of the interface property, resulting on the macroscopic level - in a brittle, softening behaviour in case of weak bond and a rather ductile, hardening behavior in case of a relatively strong interface bond that is completely described by simple microslip laws. For the finite element analyses of failure behavior at the structural level, the so-called 'interface solid element' (ISE) is used to represent the cracking process. The softening behavior of ISE is governed by the crack bridging law obtained above. The implicit-explicit integration scheme is implemented to enhance the robustness of computation. Selected numerical examples demonstrate that the crack pattern as well as the structural responses under tension-dominant stress conditions can be well simulated
\end{abstract}

\section{INTRODUCTION}

Mechanical properties such as ductility, strength and load bearing capacity of cementitious materials can be considerably improved by the addition of reinforcing fibers. These reinforcing fibers improve the fracture toughness of the unreinforced cementitious matrix by arresting microcrack growth which, if unhindered, will coalesce and form a macro-crack eventually leading to material and structural failure. The strengthening mechanism is dependent on the properties of the fiber and its interaction with the matrix material characterized by the interface behavior.

In the first part of this paper, we use micromechanics to understand how the micromechanisms influence the macroscopic behavior of the FRC composite for a unidirectional fiber orientation (see Fig. 1). At the scale of the individual fibers, the local fibre stress is associated via equilibrium conditions to interfacial shear stresses, which are governed by the interface properties of the fibre-concrete interface. Depending on the overall stress state of the FRC composite, microslip at the level of the individual fibers control the microcrack opening and, indirectly, the microcrack size. Assuming that the major toughnening mechanism in FRC composites is the role of fibers in bridging Mode-I microcracks, using linear elas- 
tic fracture mechanics at this scale, the fibrebridging mechanism is characterized by an effective Mode-I stress intensity factor [11, 15]. The homogenized macroscopic behavior of the FRC composite can be obtained using continuum micromechanics [3, 5,7, 16].

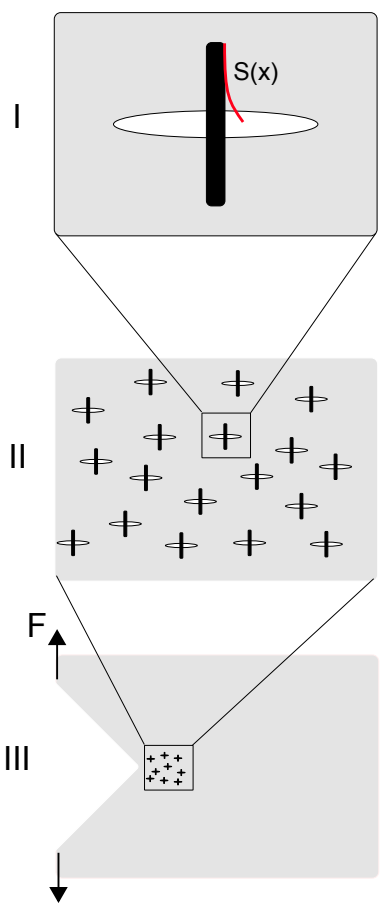

Figure 1: A multiscale approach to FRC. I: LEFM Model for microcrack growth and fibre-microslip, II: Homogenization model with unidirectional fibers bridging orthogonal microcracks, III: FRC composite structure under tensile load

In the second part of this paper, we model FRC failure at the level of the structure. For the failure analyses of structures made of FRC, most of the existing numerical models are developed based on the models for plain concrete, by means of modifying the post peak regime of inelastic constitutive models to represent the enhanced ductility of FRC in terms of an increase of the fracture energy, see e.g. [1]. These models include the effect of fibers on a phenomenological level, provided that the enhanced fracture energy of the specific fiber cocktail and the specific concrete material is known a priori from experiments.

Recently, [6] introduced a modification of the classical zero-thickness interface elements by using degenerated solid finite elements with almost zero thickness. This technique can be easily implemented in standard FE programs using conventional solid elements and has been successfully used in capturing complex cracking processes in the structures made of plainas well as reinforced concrete, without the need of any crack tracking algorithm. In the present work, this method is selected and enriched by a crack bridging model obtained from the lower scale analyses of FRC composite in order to conduct numerical simulation of structures made of FRC.

\section{MICROMECHANICS OF FRC COM- POSITES}

\subsection{Microslip Model}

Consider an FRC composite made up of a bundle of short-fibers of length $L$, all oriented in parallel and embedded in an intact porous cementitious matrix material. The stresses from the matrix is transferred to each individual fiber through the fiber-matrix interface. The stresses $\sigma_{f}$ in the fiber can be related to the shear stress $\tau$ at the fiber surface in terms of the geometrical parameters of the fiber ( $p$ is the perimeter of the fibre and $A_{f}$ is the sectional area of the fiber) using the principle of equilibrium as follows:

$$
d \sigma_{f}+\tau(y) p A_{f}^{-1} d y=0 .
$$

The shear stresses along the surface of the fiber is a function of the fiber-matrix mismatch that is a result of microslipping. This can be described by the expression $\tau(S(y))=k S(y)$. Generally, the parameter $k$ is a function of the microslip $S$ but for the time being it is assumed to be a constant. The microslip strain $S^{\prime}$ can be related to the matrix strain $\varepsilon_{m}$ and the fiber strain $\varepsilon_{f}$ through the following definition:

$$
S^{\prime}=\varepsilon_{m}-\varepsilon_{f}=\frac{\sigma_{m}}{E_{m}}-\frac{\sigma_{f}}{E_{f}}
$$

Let $f$ be the area fraction of the fibers, then, the matrix strains can be related to the far-field stresses from the expression $\Sigma=f \sigma_{f}+(1-$ f) $\sigma_{m}$. Using the following re-parametrization, $\eta=\frac{E_{f} f_{f}}{E_{m}(1-f)}, 2$ is obtained in terms of the farfield stresses and the overall fiber-stress $\Sigma_{f}=$ 
$\sigma_{f} f$ as:

$$
S^{\prime}=\frac{\eta}{E_{f} f} \Sigma^{\infty}-\frac{1+\eta}{E_{f} f} \Sigma_{f}(y)
$$

To obtain an expression for $\Sigma_{f}$ from (3), we need to compute $S^{\prime}$. This can be achieved by the following steps.

1. Differentiate (3) and use (1) to obtain the following second order differential equation for the slip along the fiber axis:

$$
S^{\prime \prime}=\frac{1+\eta}{E_{f} A_{f}} \tau(y) p
$$

2. Solve (4) and estimate the constants of integration by assuming the fiber tips are stress free $\left(\Sigma^{f}(L)=0\right)$ and that the fiber slip at the origin $y=0$ is the half crack mouth opening $S(0)=w$.

We assume for simplicity that the half crack mouth opening $w$ is related to the crack radius $a$ through the aspect ratio, $X$ of the microcrack as $w=X a$. The aspect ratio is constant during crack growth. This is a reasonable assumption for brittle microfracturing. The total stress in a short fiber bundle that is bridging a crack with a half-crack mouth opening $w$ can be adequately characterized by the geometry of the fiber, the material properties of the fiber and the matrix material and the far-field applied stress.

$$
\Sigma_{f}=g\left(\Sigma^{\infty}, w, k, b, E_{f}, E_{m}, f, L\right)
$$

According to (5), the interface behavior between the fiber and the matrix material is described by a simple linear relation $\tau(S)=k S$. This assumption cannot model softening and slip-out of the fiber. According to this assumption, as the microslip increases, the stresses in the fiber increase without bound which is not physical. Incorporating a realistic law is possible but it is not easy to extract an analytical expression analogous to (5) due to the nonlinearity. However, this effect can be incorporated using an iterated approximation of $k$ during the simulation. The shear stress is first computed explicity from a realistic microslip law $\tau^{r}(S)$ (see Fig.2) at load step $n$ given the half microcrack width $w^{(n)}$, then the parameter $k^{(n+1)}$ for the next load step is estimated from $k^{(n+1)}=\frac{\tau^{r}\left(w^{(n)}\right)}{w^{(n)}}$.

\subsection{Microcrack arrest}

The Mode-I stress intensity factor for a penny-shaped microcrack of radius $a$ bridged by fibers with stresses $\Sigma^{f}$ can be computed from:

$$
K_{I}^{*}=\frac{2}{\sqrt{\pi a}} \int_{0}^{a} \frac{\left(\Sigma^{\infty}-\Sigma_{f}\right) x}{\sqrt{a^{2}-x^{2}}} d x
$$

The material resistance against crack propagation in tension can be expressed in terms of the fracture toughness $K_{I c}$ (local stress criterion). The fracture toughness can be used as a standard to which the stress intensity factor can be compared. Microcrack growth begins if

$$
K_{I}^{*}=K_{I c}
$$

and stable microcracking occurs if:

$$
d K_{I}^{*}=0, \quad d a>0
$$

According to (6), the fiber stresses $\Sigma_{f}$ at the surface of the microcrack reduces the stress intensity factor, toughening the FRC composite.

\subsection{Homogenization}

The macroscopic stress state $\boldsymbol{\Sigma}$ due to a strain $\mathbf{E}$ in the composite following a multilevel 2-step homogenization scheme assuming the overall REV stress is equal to the far-field stress $\left(\Sigma=\Sigma^{\infty}\right.$ (see. [9])) can be written as:

$$
\boldsymbol{\Sigma}=\mathbf{C}_{I}:\left(\mathbf{I}-d \mathcal{A}_{d}\right): \mathbf{E}+d \Sigma_{f} \mathbf{1}: \mathcal{A}_{d}
$$

$\mathrm{C}_{I}$ is the homogenized elasticity tensor of the intact FRC composite without any microcracks (see [13]). $d$ is the volume fraction of microcracks and $\mathcal{A}_{d}$ is the localization tensor associated with the microcrack distribution. It can be seen that according to (9), the overall failure of the FRC composite is governed by a 'TERZHAGI -like' effective stress 
$\left(\Sigma-d \Sigma_{f} 1: \mathcal{A}_{d}\right)$ purely from micromechanical arguments [2, 4]. In this case the porepressure analog is the fiber bridging stress in the microcrack. According (9), the microcrack volume fraction is $d=N V_{c}(a, X)$, where $N$ is the microcrack density and $V_{c}=\frac{4}{3} \pi a^{3} X$ is the volume of a single microcrack. While (7) characterizes the microcrack size $a$, we require an additional assumption regarding the evolution of the microcrack density to complete (9). To this end, we assume that for a particular macroscopic strain of the FRC composite, the microcrack volume fraction for plain concrete and that of the FRC composite is the same, however, the microcrack densities and the microcrack size are completely different. According to this assumption, given the state of damage that is described by the microcrack volume fraction for plain concrete $d^{p}$, the microcrack density $N$ is obtained from the following expression:

$$
N=\frac{d^{p}}{V_{c}(a)}
$$

Thus, for the same state of damage, the FRC composite has a higher density of microcracks of smaller size in comparison to that of plain concrete that has a lower density of larger microcracks [14].

\subsection{Modeling strain hardening and strain softening FRC composites using mi- croslip laws}

In this section we show that by specifying the microslip at the fiber-matrix interface, it is possible to model a wide range of macroscopic behaviour that is consistent with the physical assumptions at the microscopic scale. For this analysis, we consider the following slip law $\tau=$ $e \tau^{\max } \Lambda^{-1} \ln \Lambda$, with $e$ denoting the exponential constant, $\tau^{\max }$ the maximum shear stress in the fiber, and $\Lambda=(e-1)\left(\frac{w}{w_{c r}}\right)+1$. Here, $w_{c r}$ is the critical half-crack width beyond which fiber starts slipping out. Fig 2 illustrates this law for $\tau^{\max }=4[M P a]$ and for two different $w_{c r}$. The interface bond properties completely characterizes both $w_{c r}$ and $\tau^{\max }$.

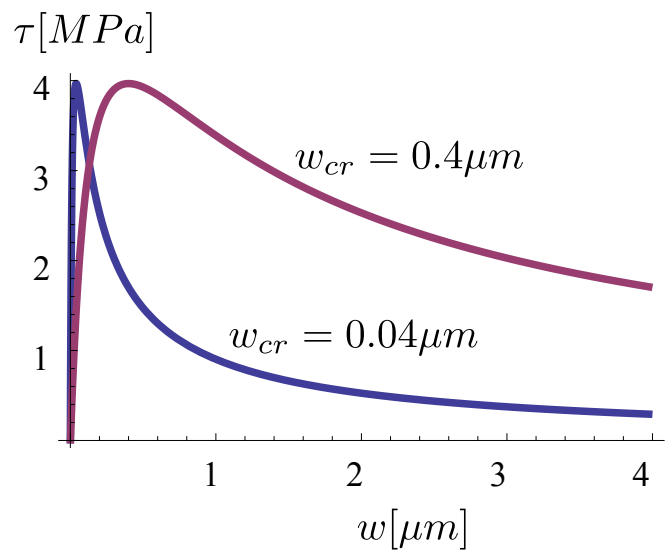

Figure 2: Microslip laws characterized by the critical microslip associated with the half microcrack width $w_{c r}$ for the same shear strength of $4[\mathrm{MPa}]$

\begin{tabular}{|c|c|}
\hline Property & Value \\
\hline \hline$K_{I C}$ & $50 \mathrm{MPa}$ \\
\hline$E_{m}$ & $50000 \mathrm{MPa}$ \\
\hline$E_{f}$ & $210000 \mathrm{MPa}$ \\
\hline$f$ & 0.01 and 0.02 \\
\hline$b$ & $0.019 \mathrm{~mm}$ \\
\hline$L$ & $13 \mathrm{~mm}$ \\
\hline
\end{tabular}

Table 1: Micromechanical material and geometrical properties of the fiber and the matrix and the volume fraction of fibers

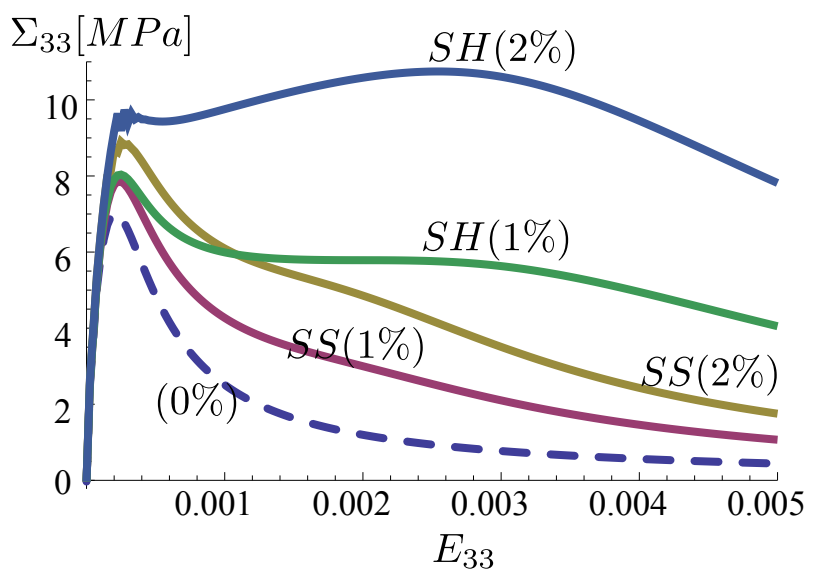

Figure 3: Macroscopic behavior of FRC composites for different fiber volume fractions $(0 \%, 1 \%$ and $2 \%)$ and two different microslip laws that show a strain-softening behavior $(S S)$ for $w_{c r}=0.04[\mu m]$ and a strain hardening behavior $(S H)$ for $w_{c r}=0.4[\mu m]$.

Fig 3, shows the macroscopic behavior of an FRC composite using the microslip laws ac- 
cording to Fig. 2 for two different fiber volume fractions ( $1 \%$ and $2 \%$ ). The material parameters used in the simulation are listed in Tab, 1 . In Fig.3, the dashed curve refers to the behaviour of plain concrete. Depending of the physics of microslip described by the microslip laws in Fig 2, the FRC composite shows a strain softening and strain hardening behavior. Evidently, the fiber content amplifies fiber induced toughening of the composite. This analysis shows that the interface shear-strength is not the only factor that determines FRC toughening but the microslip evolution. We also gain insight into the determining micromechanisms and micromechanical parameters that is invaluable in material design.

\section{FRC MODEI AT THE STRUCTURAL LEVEL}

At the structural level, a discrete representation of cracks using cohesive interface elements in conjunction with a crack bridging model is chosen. Degenerated 2D and 3D solid elements with almost zero thickness as proposed by [6, 12] are used. The formulation is based upon the principles of the continuum strong discontinuity approach for the representation of cracks in finite elements, making use of the strong discontinuity kinematics in conjunction with degenerated finite elements.

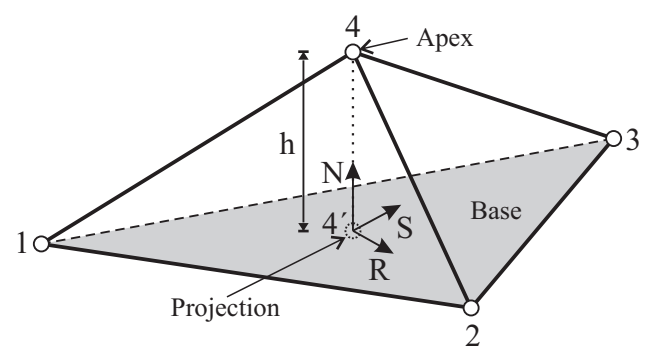

Figure 4: Degenerated 3D solid element characterized by the "base", the "apex" (Node-4) and its projection on the base (point $4^{\prime}$ ).

\subsection{Strain approximation}

Using degenerated solid elements to represent cracks, the strain in these interface solid elements is almost exclusively related to the (regularized) unbounded term $\hat{\varepsilon}$ :

$$
\boldsymbol{\varepsilon} \approx \hat{\varepsilon}=\frac{1}{h}(\llbracket \mathbf{u} \rrbracket \otimes \mathbf{n})^{s} .
$$

The displacement jump $\llbracket \mathbf{u} \rrbracket$ is determined from the relative displacement of the apex node with respect to its projection on the base (Fig. 4).

\subsection{Interface material model}

All bulk elements are considered to be linear elastic. The inelastic constitutive behavior of ISE is formulated as:

$$
\boldsymbol{\sigma}=(1-d) \mathbf{C}_{e} \boldsymbol{\varepsilon} \approx(1-d) \frac{1}{h} \mathbf{C}_{e}(\llbracket \mathbf{u} \rrbracket \otimes \mathbf{n})^{s},
$$

with $d$ as the scalar damage variable and $\mathbf{C}_{e}$ denoting the elastic stiffness matrix with the Poisson's ratio $\nu=0$.

The loading criterion is defined in terms of the equivalent stress $\tilde{\sigma}$ and the displacementlike internal parameter $\alpha$ :

$$
f(\boldsymbol{\sigma}, \alpha)=\tilde{\sigma}-t(\alpha) \leq 0 .
$$

Here, $\tilde{\sigma}=\sqrt{\sigma_{n}^{2}+\sigma_{r}^{2}+\sigma_{s}^{2}}$ is considered.

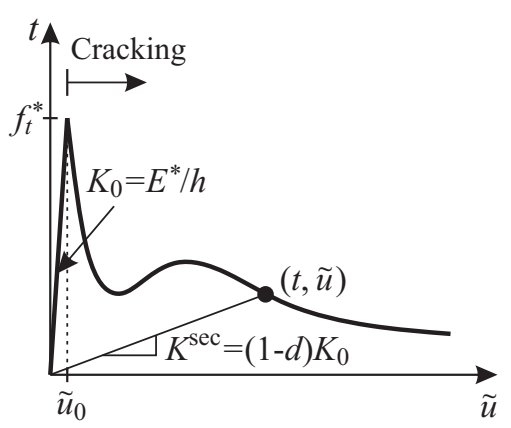

Figure 5: Equivalent traction-separation relation for the cohesive solid interface model.

The softening behavior of interface (see Fig. 5) is determined based on the parameterized function form obtained from a so-called "crack bridging model":

$$
\begin{aligned}
t(\alpha)= & \left(f_{t}^{*}-t_{1}\right) \exp \left(-\frac{\alpha}{w_{\text {ref }}}\right)+t_{1} \frac{w_{u}-\alpha}{w_{u}} \\
& +t_{2} \exp \left(c_{1}-c_{2} \alpha\right) \alpha,
\end{aligned}
$$


with $f_{t}^{*}$ the tensile strength of FRC composite and the remaining parameters appropriately determined according to the numerical results of crack bridging response obtained for the specific fiber cocktail. The crack bridging model is proposed so that, using an analytical model predicting the pullout force-displacement relation of single fibers [17] and taking an anisotropic fiber orientation into account, the integration of the pullout response of all the fibers intercepting the crack provides the traction-separation relation for an open crack. Details are included in a separate publication [18].

The internal parameter $\alpha$ is defined based on the maximum value of equivalent separation experienced during the loading history as

$$
\alpha=\max (\tilde{u})-\tilde{u}_{0} .
$$

The equivalent crack separation is defined as

$$
\tilde{u}=\sqrt{\llbracket u \rrbracket_{n}^{2}+\left(\frac{\llbracket u \rrbracket_{r}}{2}\right)^{2}+\left(\frac{\llbracket u \rrbracket_{s}}{2}\right)^{2}},
$$

and $\tilde{u}_{0}$ corresponds to the limit state of the elastic interface:

$$
\tilde{u}_{0}=\frac{f_{t}^{*}}{K_{0}}=\frac{h f_{t}^{*}}{E^{*}} \approx 0,
$$

with $K_{0}$ representing the "rigid" elastic stiffness of the equivalent interface behavior (Fig. 5).

The scalar damage variable $d(\alpha)$ is obtained by comparing the secant stiffness $K^{\mathrm{sec}}$ with the elastic stiffness of the equivalent interface behavior (Fig. 5) as

$$
d(\alpha)=1-\frac{K^{\mathrm{sec}}}{K_{0}}=1-\frac{h t}{E^{*}\left(\alpha+\tilde{u}_{0}\right)} .
$$

\subsection{Algorithmic aspects}

To enhance the robustness of computational failure analyses of FRC structures the implicitexplict (IMPL-EX) integration scheme, which combines the advantages of explicit and implicit schemes [8], is employed in the present work. The computation does not require any iteration, neither on the structural level nor on the constitutive level. This ensures the robustness and efficiency of the computational model in failure analyses of FRC structures even in cases of complex crack configurations.

\subsection{Application to numerical simulation of FRC structures}

In this section, the performance of the macroscopic model for FRC structures is demonstrated by means of re-analyses of a tension and a bending test.
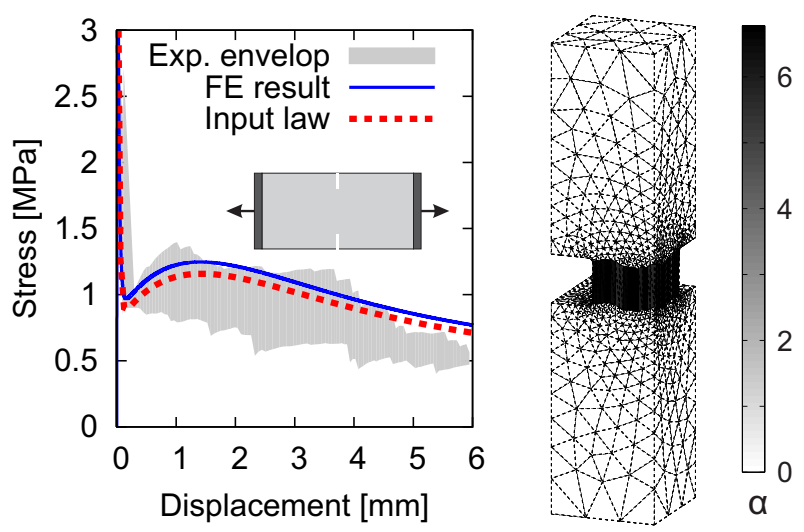

Figure 6: Analysis of a notched prism under tension: Comparison between the analytical $t(w)$ crack bridging law, the result from the finite element analysis using interface solid elements and the experimental results (left); deformation (scale=5) and contour plot of the crack opening magnitude (right).

\subsubsection{Numerical analysis of a notched FRC prism subjected to tension}

The first validation example is concerned with the uniaxial tension tests performed on notched prismatic specimens as reported in [10]. The prism has the dimension of $150 \times 150 \times 300(\mathrm{~mm})$ with a $30 \mathrm{~mm}$ notch at the middle height on each of the four faces. When subjected to a tensile force $F$, a crack develops at the notched cross section, emanating from the notches. The FRC composite is made of high strength concrete $\left(f_{c}=84 \mathrm{MPa}\right)$ and contains $0.77 \%\left(60 \mathrm{~kg} / \mathrm{m}^{3}\right)$ hooked-end steel fibers (Dramix ${ }^{\circledR}$ RC-80/60-BN).

The test is analyzed by means of a finite element simulation. Considering the symmetry of problem, 1/4 of the structure is simulated. The softening behavior of every ISE is controlled by the traction-separation law obtained from the crack bridging model, providing the 
coefficients $t_{1}=0.77, t_{2}=0.45, c_{1}=0.50$ and $c_{2}=0.64$ for the interface softening law Eq. (14). As shown in Fig. 6, the horizontal major crack develops at the middle-height (almost) instantaneously and opens in a homogeneous manner, connected with a ductile postcracking response. As expected, the finite element result of the effective stress vs. the crack mouth opening displacement relation (the blue solid curve in the diagram) is nearly identical to the input $t(\alpha)$ law (the red dashed curve), and both curves fit the experimental range very well.

\subsubsection{Numerical analysis of a notched SFRC beam}

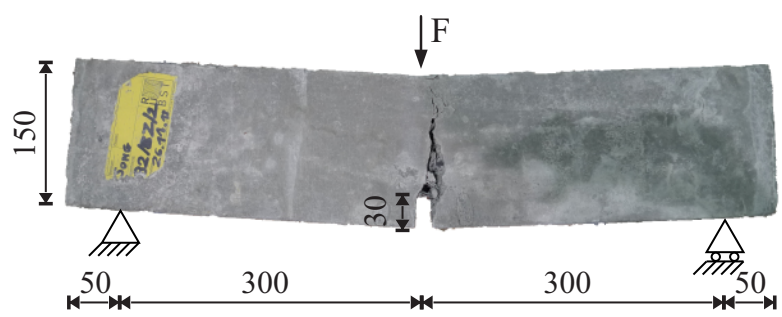

Figure 7: Analysis of a three-point bending test on a notched FRC beam: Geometry (in $\mathrm{mm}$ ) and photo of the failure state of the specimen.

The results from the laboratory tests on a series of notched beams made of different SFRC mixtures subjected to three-point bending is reported in [10]. The beam has a width of 150 $\mathrm{mm}$. A notch with a depth of $30 \mathrm{~mm}$ is located at the bottom side at the mid-span of the beam (Fig. 7). The specimens are made of high strength concrete and long steel fibers with hooked ends which are identical to the case of notched prism discussed above. In addition, short straight "micro" fibers with length 0.13 $\mathrm{mm}$, diameter $0.19 \mathrm{~mm}$ and yield stress 2200 $\mathrm{MPa}$ are used. Three different fiber cocktails are tested:

- "L60": FRC containing $60 \mathrm{~kg} / \mathrm{m}^{3}$ long hooked-end fibers,

- "S60": FRC with $60 \mathrm{~kg} / \mathrm{m}^{3}$ short straight fibers,

- "L30S30": FRC made of a mixture containing $30 \mathrm{~kg} / \mathrm{m}^{3}$ long hooked-end fibers and $30 \mathrm{~kg} / \mathrm{m}^{3}$ short straight fibers.

For the numerical simulations, the interface softening law $t(\alpha)$ characterized by $t_{1}=1.40$, $t_{2}=1.10, c_{1}=1.05$ and $c_{2}=0.78$ for the FRC design L60 and $t_{1}=1.04, t_{2}=c_{1}=c_{2}=0$ for the FRC cocktail $\mathrm{S} 60$ is used. For the composite L30S30, the traction separation law is assumed as the weighted average of the previous two.

(a)

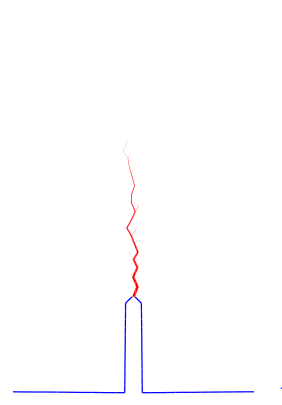

(b)

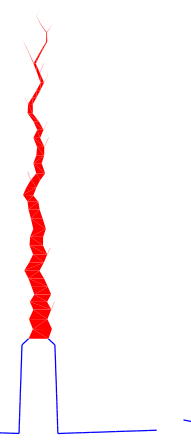

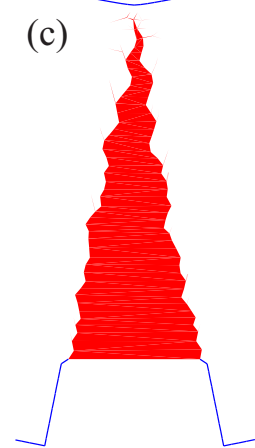

Figure 8: Numerical analysis of a notched beam subjected to three-point bending (Case-L60): The crack pattern represented by the activated interface solid elements during the loading procedure (red areas, with deformation scale $=25$ ) at the displacement level of (a) $0.06 \mathrm{~mm}$, (b) $0.32 \mathrm{~mm}$ and (c) $2.24 \mathrm{~mm}$.

In all three cases, the major crack initiates at the notch-tip and propagates vertically until reaching the top surface. Fig. 8 show, that this cracking process is well represented by the activated interface solid elements. Fig. 9 contains the force versus crack mouth opening displacement relations for the three fiber cocktails obtained from the finite element model and comparisons with the experimental results. In general, the structural response is nearly identical for all the three cases until the first peak of reaction force. Afterwards, the structure exhibits a ductile behavior with remarkable residual loading capacity, as the crack opens and the fibers bridging the crack are successively activated and pulled out from the concrete matrix. 
It is noted, that the main load carrying mechanisms of all three tests with three different fiber cocktails are replicated remarkably well, both regarding the qualitative shape of the loaddisplacement curve and quantitatively.

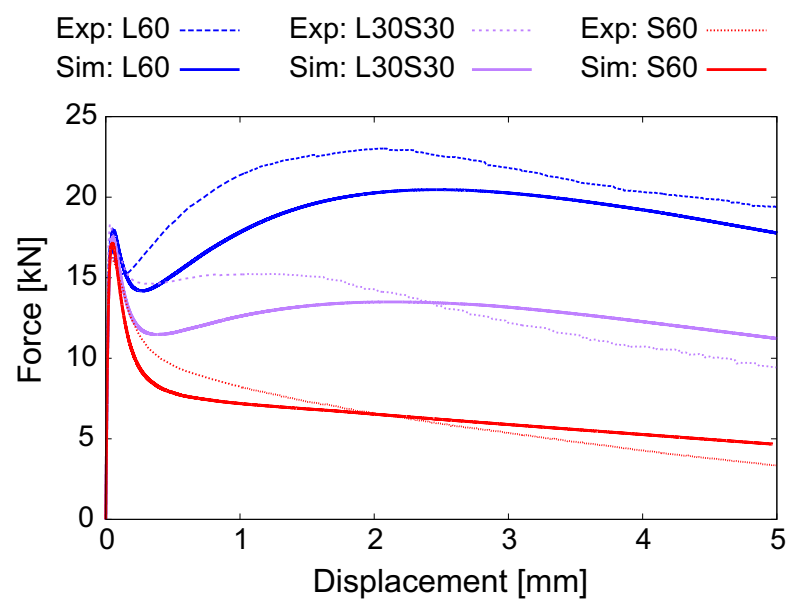

Figure 9: Numerical analysis of a notched beam subjected to three-point bending: Comparison between force-crack mouth opening displacement relations obtained from the simulations and from the experiments for three different fiber cocktails.

\section{CONCLUSIONS}

To support material design and understand the various aspects of the FRC composite, we have investigated multiscale models characterizing FRC composites at multiple scales. A multiscale continuum modeling approach based upon a combination of LEFM and continuum micromechanics was proposed for fiber reinforced cementitious composites. Using micromechanical analyses, we have shown that the overall deformation of the FRC is governed by a 'TERZHAGI' like effective stress. By specifying the microslip physics at the scale of the fiber, the homogenized macroscopic response can be tailored to a specific application ranging from a strain softening FRC composite to a strain hardening high performance FRC composites. At the level of structural finite element analyses, to represent the opening and propagation of cracks, interface solid elements have been supplied with the crack bridging model as traction-separation law. One advantage of this method is, that it does not require specific crack tracking techniques. The implicit/explicit integration scheme is implemented for the integration of the (highly nonlinear) traction separation model. This strategy has proven as very efficient as in each load increment only a linear system has to be solved without iterations.

\section{REFERENCES}

[1] E. Denneman, R. Wu, E. P. Kearsley, and A. T. Visser. Discrete fracture in high performance fibre reinforced concrete materials. Engineering Fracture Mechanics, 78(10):2235-2245, 2011.

[2] L. Dormieux, D. Kondo, and F.J. Ulm. Microporomechanics. Wiley \& Sons, 2006.

[3] Z. Hashin. Analysis of composite materials - a survey. Journal of Applied Mechanics, 50(3):481-505, 1983.

[4] C. Hellmich and F.-J. Ulm. Drained and undrained poroelastic properties of healthy and pathological bone:a poromicromechanical investigation. Transport in Porous Media, 58:243-268, 2005.

[5] $\mathrm{S}$. Li and G. Wang. Introduction to Micromechanics and Nanomechanics. World Scientific, 2008.

[6] O.Ll Manzoli, A. L. Gamino, E.A. Rodrigues, and G.K.S. Claro. Modeling of interfaces in two-dimensional problems using solid finite elements with high aspect ratio. Computers \& Structures, 94:70-82, 2012.

[7] S Nemat-Nasser and H. Hori. Micromechanics: Overall properties of heterogeneous materials. Elsevier, North- Holland, 2 edition, 1999.

[8] J. Oliver, A.E. Huespe, and J.C. Cante. An implicit/explicit integration scheme to increase computability of non-linear material and contact/friction problems. Computer Methods in Applied Mechanics and Engineering, 197(21):1865-1889, 2008. 
[9] B. Pichler, C. Hellmich, and H.A. Mang. A combined fracture-micromechanics model for tensile strain-softening in brittle materials, based on propagation of interacting microcracks. International Journal for Numerical and Analytical Methods in Geomechanics, 31(2):111-132, 2007.

[10] T. Putke, F. Song, Y. Zhan, P. Mark, R. Breitenbuecher, and G. Meschke. Development of hybrid steel-fibre reinforced concrete tunnel lining segmentsexperimental and numerical analyses from material to structural level. Bauingenieur, 89:447-456, 2014.

[11] J. Qu and M. Cherkaoui. Fundamentals of Micromechanics of Solids. John Wiley \& Sons, Inc., 2006.

[12] M. Sánchez, O.L. Manzoli, and L.J.N. Guimarães. Modeling 3-d desiccation soil crack networks using a mesh fragmentation technique. Computers and Geotechnics, 62:27-39, 2014.

[13] J. J. Timothy and G. Meschke. A cascade continuum micromechanics model for the elastic porperties of porous materials. International Journal of Solids and Structures, page 10.1016/j.ijsolstr.2015.12.010, 2016.
[14] J.J. Timothy and G. Meschke. A continuum micromechanics lefm model for fiber reinforced concrete. In G. Meschke N. Bićanić, H. Mang and R. de Borst, editors, Computational Modelling of Concrete Structures (EURO-C 2014), pages 327-332. CRC Press/Balkema, NL, 2014.

[15] K Yanase and JW Ju. Toughening behavior of unidirectional fiber reinforced composites containing a crack-like flaw: Matrix crack without fiber break. International Journal of Damage Mechanics, 22(3):336-355, 2013.

[16] A Zaoui. Continuum micromechanics: Survey. Journal of Engineering Mechanics (ASCE), 128 (8):808-816, 2002.

[17] Y. Zhan and G. Meschke. Analytical model for the pullout behavior of straight and hooked-end steel fibers. Journal of Engineering Mechanics, 140(12):04014091, 2014.

[18] Y. Zhan and G. Meschke. Failure analyses of steel fiber reinforced concrete structures in a multi-scale framework. Journal of Engineering Mechanics, page under review, 2016. 